\title{
Deconstructing the relief inversion effect: Contributors of the problem and its solutions
}

\author{
Arzu Çöltekin a,b *, Gianna Hartung a, Martina Meyer ${ }^{\mathrm{a}}$ \\ ${ }^{a}$ Department of Geography, University of Zurich, <arzu.coltekin@geo.uzh.ch>, Gianna Hartung < giannahartung@gmx.ch>, \\ Martina Meyer <meyer_martina@gmx.ch> \\ ${ }^{b}$ Institute for Interactive Technologies, FHNW University of Applied Sciences and Arts Northwestern Switzerland, \\ $<$ arzu.coltekin@fhnw.ch> \\ * Corresponding author
}

Keywords: Terrain representations, satellite imagery, relief inversion effect, terrain reversal effect

Terrain reversal (also known as relief inversion) effect is a common and well-known illusion encountered in shaded relief maps and satellite imagery where the main depth cue is shading/shadows (Imhof, 1967; Bernabe-Poveda, Callejo, \& Ballari, 2005; Saraf, Das, Agarwal, \& Sundaram, 1996; Biland \& Çöltekin, 2016; Çöltekin, Rautenbach, Coetzee, \& Mokwena, 2018). This illusion interferes with our perception of shape from shading (e.g., see Kleffner \& Ramachandran, 1992; Prados \& Faugeras, 2006). If the light shines from below, the shadows are then above, and this conflicts with the human mind's 'unconscious statistics', that is, our minds assume that the light is more or less always above. This cognitive phenomenon is termed light from above prior (Kleffner \& Ramachandran, 1992). When the prior is violated, we see three-dimensional (3D) shapes ambiguously, or inverted; such that a valley looks like a ridge in a terrain representation and vice versa (Bernabé-Poveda, Sánchez-Ortega, \& Çöltekin, 2011; Bernabé-Poveda \& Çöltekin, 2014). In a recent study, we demonstrated that adding texture and color as opposed to the shading alone (as in the shaded relief maps) affect 3D shape identification performance (Çöltekin \& Biland, 2018). More precisely, when texture is present, success rates are higher in correctly identifying valleys and ridges, as well as other 3D spatial relationships. This is possibly a result of interpreting the spatial relationships between terrain features because people can recognize them more easily (e.g., a river is easier to identify on a photo than on a shaded relief map). Somewhat surprisingly, we also observed that people are better with 3D shape identification with grayscale images than with the color images; which we interpreted as the result of more pronounced contrast in grayscale images (Çöltekin \& Biland, 2018). Because we see that presence of other visual cues do interfere with the relief inversion effect, in this study, we explore other additional factors (depth cues, labels, terrain types, task types, expertise levels and spatial abilities) that may contribute to, or alleviate, the relief inversion effect. Understanding how other factors contribute to the strength of the illusion can help develop better-informed use of the displays that contain this illusion and lead to better solutions. In this vein, in a series of experiments, we examined effects of stereoscopic viewing vs. monoscopic viewing, terrain types (highly rugged vs. subtly rugged), task types (3D shape identification vs. land cover identification; simple vs. complex), expertise levels, and spatial abilities. In a second experiment, we examined how well various solutions function for correcting the illusion in various combinations of variables (labels, motion, stereo). Figure 1 shows an example of the shape identification tasks used in the first study.
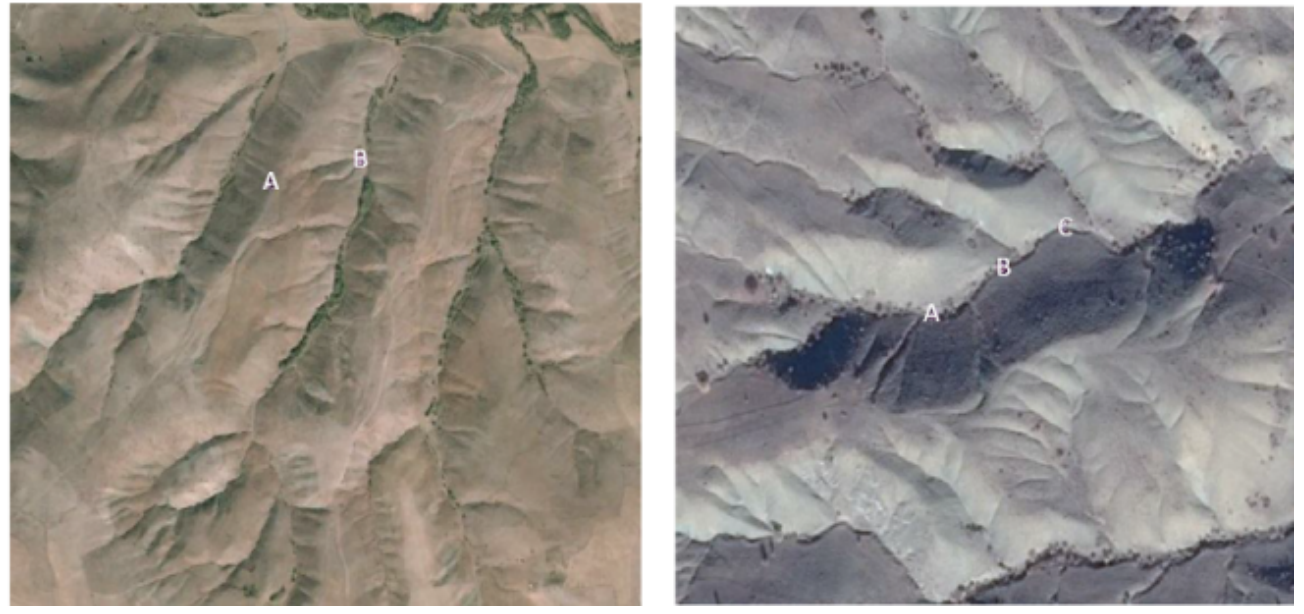

Figure 1. On the left, participants were asked to identify is the point labelled A was on a higher elevation than B. On the right, they were asked if they see a valley or a ridge. Both images contain the terrain reversal effect, that is, the answers are "A is higher than B" for the left image, and "valley" for the right image. Same images were also shown in anaglyph stereo and same questions were asked. Participants worked with 240 items in total in a randomized manner. Images are sourced from Google Earth and labels were added by the project team. 
We are developing full papers reporting the effects of each tested factor in detail, which would be beyond the scope of this short paper. In this short paper, we focus on the effects of stereo on the strength of the relief inversion experience (does showing the terrain in stereo make the relief inversion effect stronger or weaker?), and on the solutions for satellite images (if we combine a 'solution' with stereo viewing, do shape perception and land cover identification success improve?). The second question, especially the mention of land cover identification, is related to the fact that a common solution to terrain reversal effect is to overlay a semi-transparent shaded relief map (SRM overlay) on top of the image that has the perceptual problem (Bernabé-Poveda, Sánchez-Ortega, \& Çöltekin, 2011; Saraf, Das, Agarwal, \& Sundaram, 1996b).

Our initial findings suggest that stereo viewing does help against the issues in 3D shape perception; although it does not entirely remove it: $3 \mathrm{D}$ shape perception accuracy is $\sim 15 \%$ with original images, $\sim 32 \%$ with the stereo. When stereoscopic viewing is combined with an SRM overlay solution, it improves the 3D shape perception: 3D shape perception accuracy with the SRM overlay solution alone is $\sim 40 \%$, with added stereo $\sim 68 \%$, but impairs the land cover identification (accuracy is $\sim 78 \%$ with the SRM overlay, and drops to $\sim 44 \%$ when stereo is added). These findings are based on two controlled experiments with 33 and 35 participants respectively, and the differences are statistically significant based on analysis of variance $(\mathrm{p}<.05)$. We believe the impairment of the land cover identification is linked to the stereoscopic viewing method, as the tests were conducted with anaglyph stereo, where color perception is strongly affected. These observations, taken together, provide us the initial clues that providing the viewers with an additional depth cue (in this case stereopsis) is indeed helpful; but also suggest that the success of the solution depends on how it is implemented, and the nature of the task; for example, if color is important for the task or not.

We believe our findings are of key importance in understanding the relief inversion effect, and its future solutions, and will guide cartographers towards a more nuanced comprehension and work practices.

\section{References}

Bernabé-Poveda, M. a., Sánchez-Ortega, I., \& Çöltekin, A. (2011). Techniques for highlighting relief on orthoimaginery. Procedia - Social and Behavioral Sciences, 21, 346-352. http://doi.org/10.1016/j.sbspro.2011.07.028

Bernabé-Poveda, M. A., \& Çöltekin, A. (2014). Prevalence of the terrain reversal effect in satellite imagery. International Journal of Digital Earth, 1-24. http://doi.org/10.1080/17538947.2014.942714

Biland, J., \& Çöltekin, A. (2016). An empirical assessment of the impact of the light direction on the relief inversion effect in shaded relief maps: NNW is better than NW. Cartography and Geographic Information Science, 1-15. http://doi.org/10.1080/15230406.2016.1185647

Çöltekin, A., \& Biland, J. (2018). Comparing the terrain reversal effect in satellite images and in shaded relief maps: an examination of the effects of color and texture on 3D shape perception from shading. International Journal of Digital Earth, 1-18. http://doi.org/10.1080/17538947.2018.1447030

Çöltekin, A., Rautenbach, V., Coetzee, S., \& Mokwena, T. (2018). Landform perception accuracy in shaded relief maps: a replication study confirms that nnw lighting is better than nw against the relief inversion effect. ISPRS International Archives of the Photogrammetry, Remote Sensing and Spatial Information Sciences, XLII-4, 101106. http://doi.org/10.5194/isprs-archives-XLII-4-101-2018

Imhof, E. (1967). Shading and Shadows. Chapter 9. Cartographic Relief Representation (Vol. 2007, pp. 159-212).

Kleffner, D. a, \& Ramachandran, V. S. (1992). On the perception of shape from shading. Perception \& Psychophysics, 52(1), 18-36. Retrieved from http://www.ncbi.nlm.nih.gov/pubmed/1635855

Bernabé-Poveda, M., Callejo, M., \& Ballari, D. (2005). Correction of Relief Inversion in Images Served by a Web Map Server. In: The Proceedings of the $22^{\text {nd }}$ International Cartograhic Conference (ICC) (Vol. 1). A Coruña, Spain, 9-16 July 2005.

Prados, E., \& Faugeras, O. (2006). Shape from shading. In Handbook of mathematical models in computer ... (pp. 375388). http://doi.org/10.1007/0-387-28831-7_23

Saraf, A. K., Das, J. D., Agarwal, B., \& Sundaram, R. M. (1996). False topography perception phenomena and its correction. International Journal of Remote Sensing, 17(18), 3725-3733. http://doi.org/10.1080/01431169608949180 